\title{
polymers
}

ISSN 2073-4360

www.mdpi.com/journal/polymers

Article

\section{The Influence of Synthesis Parameters on Particle Size and Photoluminescence Characteristics of Ligand Capped $\mathbf{T b}^{3+}: \mathbf{L a F}_{3}$}

\author{
Kyle Gipson ${ }^{1}$, Courtney Kucera ${ }^{1}$, Derek Stadther ${ }^{2}$, Kathryn Stevens ${ }^{1}$, John Ballato ${ }^{1}$ and \\ Phil Brown ${ }^{1, *}$
}

1 Center for Optical Materials Science \& Engineering Technologies (COMSET), School of Materials Science and Engineering, Clemson University, Clemson, SC 29634, USA;

E-Mails: kgipson@clemson.edu (K.G.); courtnk@clemson.edu (C.K.); ksteven@clemson.edu (K.S.); jballat@ clemson.edu (J.B.)

2 Department of Physics, Furman University, Greenville, SC 29613, USA;

E-Mail: derek.stadther@furman.edu (D.S.)

* Author to whom correspondence should be addressed; E-Mail: pjb @ clemson.edu; Tel.: +1-864-656-6072; Fax: +1-864-656-5973.

Received: 11 October 2011; in revised form: 11 November 2011 / Accepted: 17 November 2011 / Published: 29 November 2011

\begin{abstract}
Organic ligand surface-treated $\mathrm{Tb}^{3+}: \mathrm{LaF}_{3}$ was synthesized in water and methanol for subsequent incorporation into polymethyl methacrylate (PMMA) via solution-precipitation chemistry in order to produce optically active polymer nanocomposites. Nanoparticle agglomerate diameters ranged from $388 \pm 188 \mathrm{~nm}$ when synthesized in water and $37 \pm 2 \mathrm{~nm}$ when synthesized in methanol. Suspension stability is paramount for producing optically transparent materials. Methanol nanoparticle synthesized at a $\mathrm{pH}$ of 3 exhibited the smallest agglomerate size. Optical spectroscopy, dynamic light scattering, transmission electron microscopy, scanning transmission electron microscopy, and zeta potential analysis were used to characterize the particles synthesized.
\end{abstract}

Keywords: methanol; nanoparticles; agglomerates; acetylsalicylic acid 


\section{Introduction}

\subsection{Optically-Active Polymer Nanocomposites}

Optically-active polymer nanocomposites are suitable materials for use in a series of optoelectronic applications due to their low cost and ease of processing [1,2]. Amorphous polymers such as polymethyl methacrylate (PMMA) are utilized in numerous optical applications because they are highly transparent in the visible. PMMA has been the most predominate polymer employed to date for optical purposes and has been shown to be a suitable medium for certain rare-earth (RE) ion ligand complexes [3] that generate light. While the higher attenuation of polymers, relative to inorganic glasses (e.g., silica optical fiber), limits their use to distances of less than about $1 \mathrm{~km}$ [4], there are still many applications for light emissive polymer fibers, films, and coatings.

Luminescent species such as RE ions have been utilized as active additives to produce emissions in the visible region of the electromagnetic spectrum for various inorganic glasses and crystals. RE ions can produce intense narrow spectral emissions with long excited-state lifetimes, in the range of milliseconds, as a result of their partially-filled and electrostatically-shielded $4 f$ electronic levels $[5,6]$. These advantageous radiative properties of rare-earth ions unfortunately tend to be quenched by the inherently high vibrational energies of polymers [7]. Additionally, RE ion salts exhibit limited solubility in polymers and these salts tend to aggregate resulting in enhanced scattering as well as luminescent concentration quenching at low concentrations [8].

Polymer nanocomposites comprised of organic ligand capped inorganic nanocrystals are studied here in order to merge the spectroscopic benefits of RE ions in low vibrational energy inorganic hosts with the processability of polymeric materials. More specifically, the function of a surface organic ligand (acetylsalicylic acid; ASA) is used to absorb and transfer UV light to RE ions that are doped into inorganic nanocrystals dispersed into PMMA. ASA is an aromatic carboxylic acid and aromatic acids have been used as ligands to coordinate, to sensitize and to enhance lanthanide fluorescence of $\mathrm{RE}$ ions [9-11]. The inorganic nanocrystal (lanthanum trifluoride; $\mathrm{LaF}_{3}$ ) provides a low phonon energy environment $\left(350 \mathrm{~cm}^{-1}\right)$ and offers channels for charge transfer excitations from near anionic ligands [12,13]. $\mathrm{LaF}_{3}$ also exhibits a high solubility for RE ions dopants of the same valence within the crystal [14]. In this work, the RE ion of choice is trivalent terbium $\left(\mathrm{Tb}^{3+}\right)$. The most intense emission band of $\mathrm{Tb}^{3+}$ from direct excitation at $350 \mathrm{~nm}$ is located at about $543 \mathrm{~nm}$ which emits green colored light that is easily distinguished visually.

Energy transfer from the ligand to the RE-doped nanocrystal begins with the excitation of the ligand, as is illustrated in Figure 1. Organic ligands typically absorb energy in the near ultraviolet (UV) spectral region ranging from $200 \mathrm{~nm}$ to about $400 \mathrm{~nm}$ [15]. Typically, singlet spin states of the ligand are excited followed by intersystem crossing from the singlet state to the lowest triplet state within the ligand through thermal relaxation accompanied by the generation of heat. The occurrence of intersystem crossing is in competition with phonon deactivation of the excited singlet state and, potentially, fluorescence from the ligand [8]. Energy transfer may then occur from the triplet state of the ligand to the RE ion when the energy of the triplet state is reasonably equivalent to that of a suitable energy level of the dopant. Light emission from the RE ion results from the radiative transition between $4 f$ states. 
Figure 1. Ligand to rare-earth (RE) ion energy transfer diagram [16].

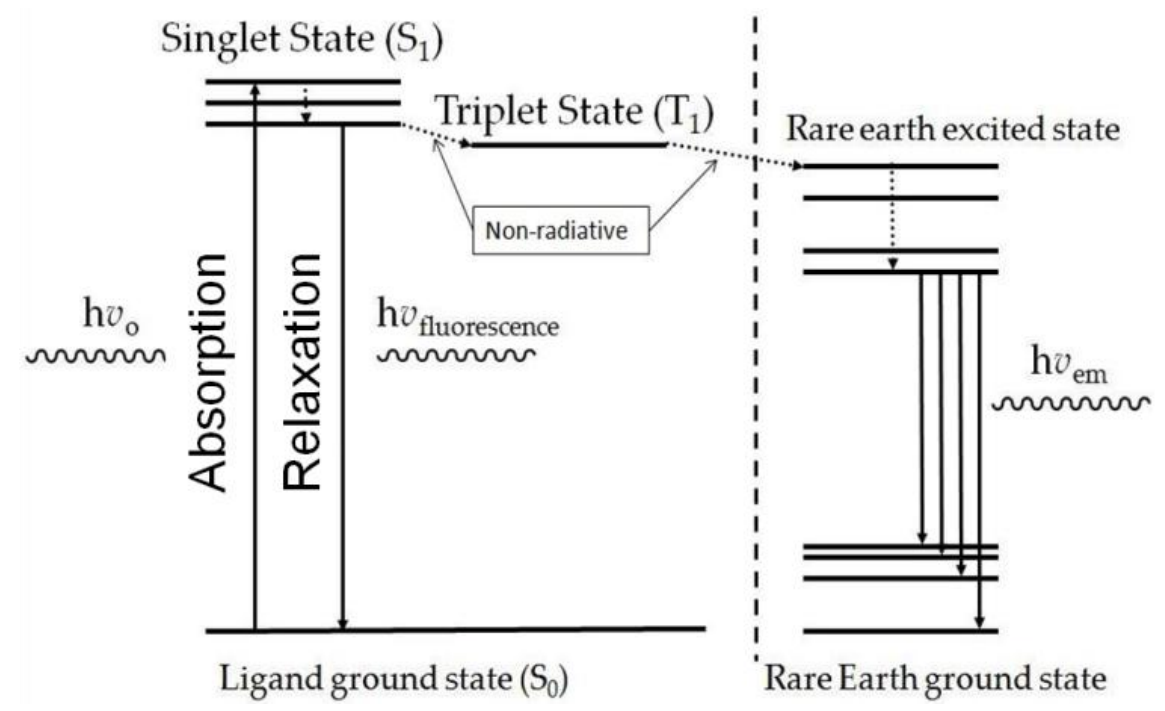

\subsection{Review of Electrical Double Layer Theory}

Nanoparticles, such as those employed here, generally refer to materials whose dimensions are within the range of 1-100 nm. At these small sizes, surface interactions strongly influence their properties [17]. The electrical double layer is a representation of the ion distribution at the interface between a solid and a solvent in solution/suspension as is illustrated in Figure 2. Helmholtz first introduced and termed the idea of the electrical double layer which was later extended by Gouy-Chapman and Stern [18-22]. The Stern layer is the first stratum that consists of a dense layer of ions of the opposite charge (counter ions) that form around the nanoparticle. The second layer is the diffuse layer which is the charged atmosphere of ions of the opposite charge to the nanoparticle that extends away from the Stern layer. The high concentration of counter ions within the diffuse layer gradually decreases with increasing distance from the nanoparticle until equilibrium is reached with the ion concentration in the bulk of the solvent [23].

Figure 2. Electrical double layer distribution of ions.

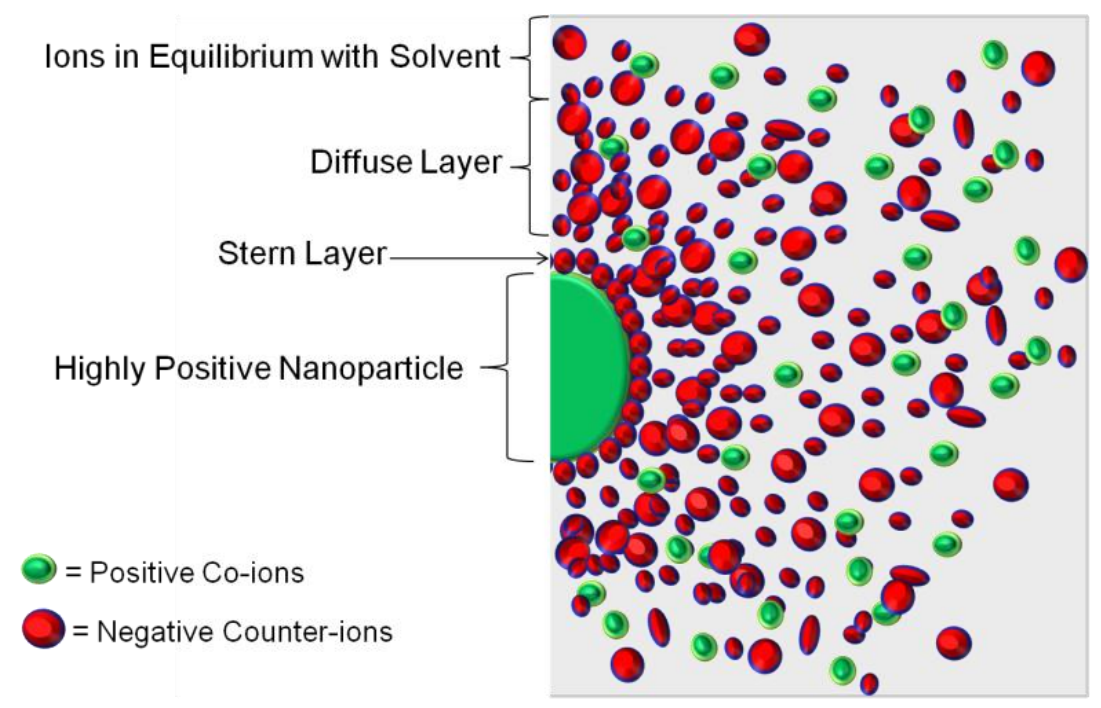


The width of the electrical double layer is characterized by the thickness of the diffuse layer or Debye screening thickness which is represented by $\kappa^{-1}$ (Equation 1). The relative thickness of the diffuse layer coincides with the ability of the nanoparticle to repel (colloidally stable) or attract other nanoparticles (agglomeration). Agglomerates form as the electrical double layer is condensed by the nanoparticles attempting to reduce its surface area to minimize interfacial energy [24]. The scattering of light will occur as agglomerate sizes increase; consequentially, diminishing the transparency of the polymer nanocomposites; which influences the ability of the RE ion to absorb and emit efficiently. Therefore, it is important that a polymer with additives be optically transparent in the region of the excitation and emission wavelengths of the RE ion [13].

$$
\kappa^{-1}=\sqrt{\frac{\varepsilon_{r} \varepsilon_{o} \mathrm{k}_{\mathrm{B}} \mathrm{T}}{2 \mathrm{~N}_{\mathrm{A}} \mathrm{e}^{2} \mathrm{I}}}
$$

$\boldsymbol{I}=$ ionic strength of the electrolyte $\left(\mathrm{mol} / \mathrm{m}^{3}\right)$

$\boldsymbol{\varepsilon}_{\mathbf{r}}=$ dielectric constant of solvent

$\boldsymbol{T}=$ absolute temperature (Kelvin)

Constants:

$\boldsymbol{\varepsilon}_{\mathbf{0}}=$ permittivity of free space $\left(8.85 \times 10^{-12} \mathrm{C}^{2} / \mathrm{Jm}\right)$

$\boldsymbol{k}_{\mathbf{B}}=$ Boltzmann constant $\left(1.38 \times 10^{-23} \mathrm{~J} / \mathrm{K}\right)$

$N_{A}=$ Avogadro number $\left(6.02 \times 10^{23} \mathrm{~mol}^{-1}\right)$

$\boldsymbol{e}=$ elementary charge $\left(1.60 \times 10^{-19} \mathrm{C}\right)$

The major contributors to the thickness of the diffuse layer are ionic strength of the electrolyte $(I)$, absolute temperature in Kelvin $(T)$ and dielectric constant of the solvent $\left(\varepsilon_{\mathrm{r}}\right)$. The ionic strength is inversely proportional to $\kappa^{-1}$. Therefore, as the ionic strength lowers the double layer thickness should increase potentially resulting in less agglomeration; hence, low optical scattering. The Debye screening thickness is directly related to the dielectric constant and temperature. Temperature and the dielectric constant of the solvent are indirectly related to each other which may lead to only a slight change in the Debye screening thickness.

\subsection{Methods of Nanoparticle Synthesis Review}

Reverse micelle synthesis, sol-gel chemistry, solvothermal, hydrothermal and aqueous solution-polymer precipitation have all been utilized to produce inorganic nanoparticles [17,25-28]. Reverse micelle synthesis require significant amounts of surfactants to produce limited amounts of nanoparticles [28]. Solvothermal and hydrothermal techniques require an autoclave at elevated temperatures and pressures. Sol-gel chemistry and solution-polymer precipitation require a drying step which may increase aggregation. In the present work, aqueous solution-polymer precipitation techniques were chosen as the result of the possibility to control size and size distribution of the nanoparticle [29]. We report on the effect of solvent relative dielectric constant, synthesis $\mathrm{pH}$, and temperature on the diameter of ASA: $\mathrm{Tb}^{3+}: \mathrm{LaF}_{3}$ nanoparticles in solution and PMMA via solution-polymer precipitation. 


\section{Results and Discussion}

Elemental composition of the nanoparticles determined by EDX analysis is summarized in Table 1. The resulting composition consisted of $\mathrm{Tb}^{3+}: \mathrm{LaF}_{3}$ at a 5:1 and 2:1 (La:Tb; i.e., $\left(\mathrm{Tb}_{0.16} \mathrm{La}_{0.84}\right) \mathrm{F}_{3}$ and $\left.\left(\mathrm{Tb}_{0.33} \mathrm{La}_{0.67}\right) \mathrm{F}_{3}\right)$ molar ratio for the nanoparticles synthesized in water and methanol solvents respectively.

Table 1. Elemental composition and corresponding atomic percentage of nanoparticles in polymethyl methacrylate (PMMA) synthesized in different solvents.

\begin{tabular}{ccc}
\cline { 2 - 3 } Element & \multicolumn{2}{c}{ Atomic Percentage of Elements in } \\
\cline { 2 - 3 } & Water Synthesis & Methanol Synthesis \\
\hline $\mathrm{La}$ & 43 & 28 \\
$\mathrm{~Tb}$ & 9 & 14 \\
$\mathrm{~F}$ & 48 & 58 \\
\hline
\end{tabular}

The main absorption peak for the ASA ligand is located at about $276 \mathrm{~nm}$ when the ligand is dispersed in water and methanol. A representative absorption spectra of the ASA ligand (blue), $\mathrm{Tb}\left(\mathrm{NO}_{3}\right)_{3} \cdot 6 \mathrm{H}_{2} \mathrm{O}$, (green), and $\mathrm{Tb}^{3+}: \mathrm{LaF}_{3}$ (red) is shown in Figure 3. This excitation wavelength was used to evaluate the energy transfer of the ligand to the RE ion. The peak value for $\mathrm{Tb}\left(\mathrm{NO}_{3}\right)_{3} \cdot 6 \mathrm{H}_{2} \mathrm{O}$ and $\mathrm{Tb}^{3+}: \mathrm{LaF}_{3}$ respectively was measured at $340 \mathrm{~nm}$.

Figure 3. Absorption spectra of acetylsalicylic acid (ASA) (blue), ASA (red) ligand, $\mathrm{Tb}\left(\mathrm{NO}_{3}\right)_{3} \cdot 6 \mathrm{H}_{2} \mathrm{O}$ (green) and $\mathrm{Tb}^{3+}: \mathrm{LaF}_{3}$ (red) in water.

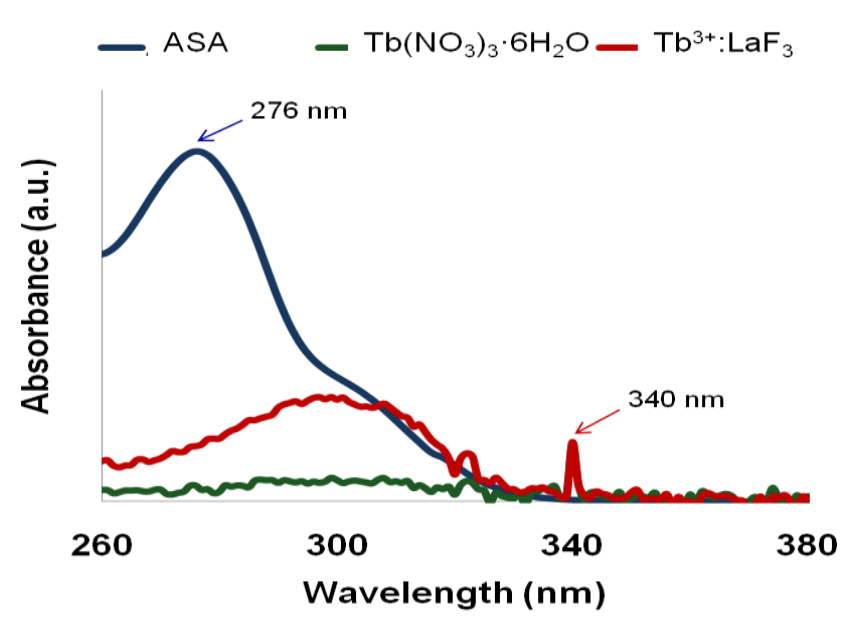

Excitation spectra of $\mathrm{Tb}^{3+}$ and ASA at the $540 \mathrm{~nm}$ emission wavelength were measured and are displayed in Figure 4. Direct excitation of $\mathrm{Tb}^{3+}(350 \mathrm{~nm})$ and ligand excitation $(276 \mathrm{~nm})$ were evaluated for each solvent as is shown in Figure 5(a,b), respectively.

The emissions spectra for the nanoparticle loaded polymer (PMMA:ASA: $\mathrm{Tb}^{3+}: \mathrm{LaF}_{3}$ ) upon direct ion and ligand excitation exhibited characteristic $\mathrm{Tb}^{3+}$ emissions at 490, 543, 585, and $621 \mathrm{~nm}$ corresponding to the ${ }^{5} \mathrm{D}_{4} \rightarrow{ }^{7} \mathrm{~F}_{6},{ }^{5} \mathrm{D}_{4} \rightarrow{ }^{7} \mathrm{~F}_{5},{ }^{5} \mathrm{D}_{4} \rightarrow{ }^{7} \mathrm{~F}_{4}$, and ${ }^{5} \mathrm{D}_{4} \rightarrow{ }^{7} \mathrm{~F}_{3}$ transitions, respectively. Energy transfer from the ligand to the $\mathrm{RE}$ ion is presumed by the excitation of the ligand and resulting characteristic emissions from the $\mathrm{Tb}^{3+}$. 
Figure 4. Excitation spectra for $\mathrm{Tb}^{3+}$ and ASA measuring the $540 \mathrm{~nm}$ peak.

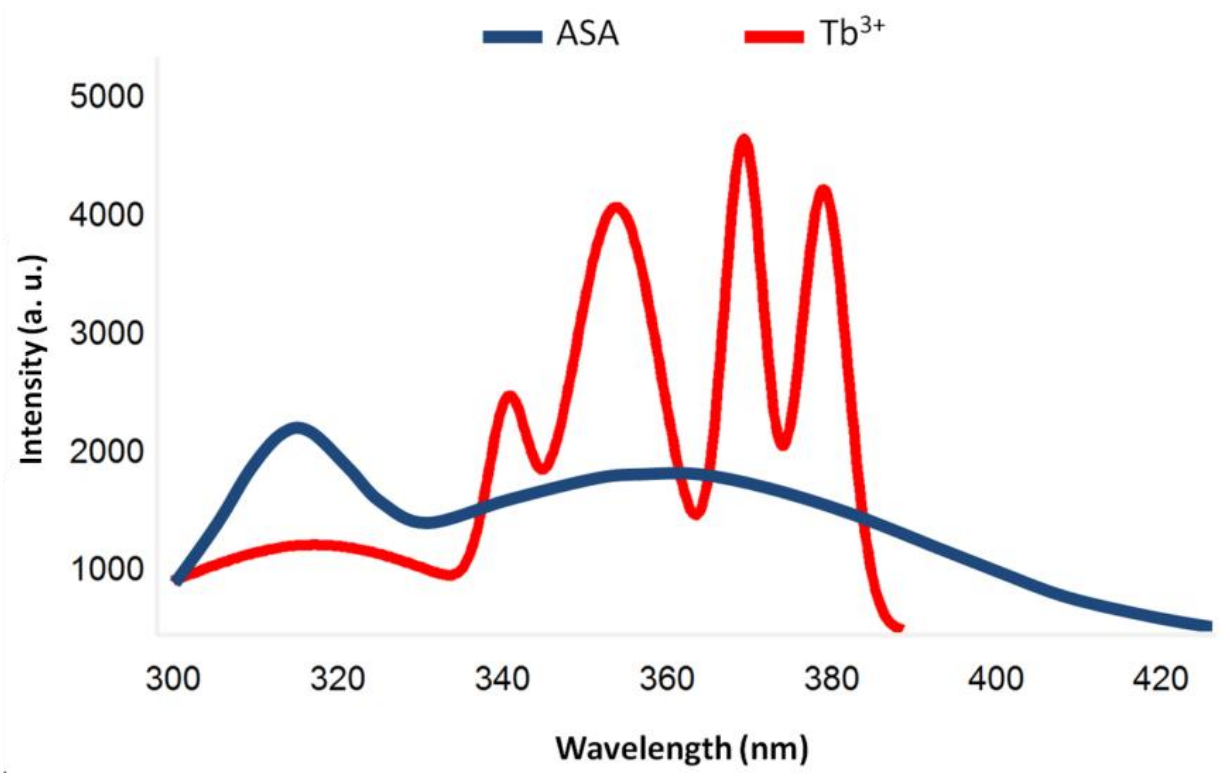

Figure 5. (a) Photoluminescence spectra under direct ion excitation at $350 \mathrm{~nm}$ of PMMA:ASA: $\mathrm{Tb}^{3+}: \mathrm{LaF}_{3}$ nanocomposite; (b) Photoluminescence spectra under direct ligand excitation at $276 \mathrm{~nm}$ of PMMA:ASA: $\mathrm{Tb}^{3+}: \mathrm{LaF}_{3}$ nanocomposite.

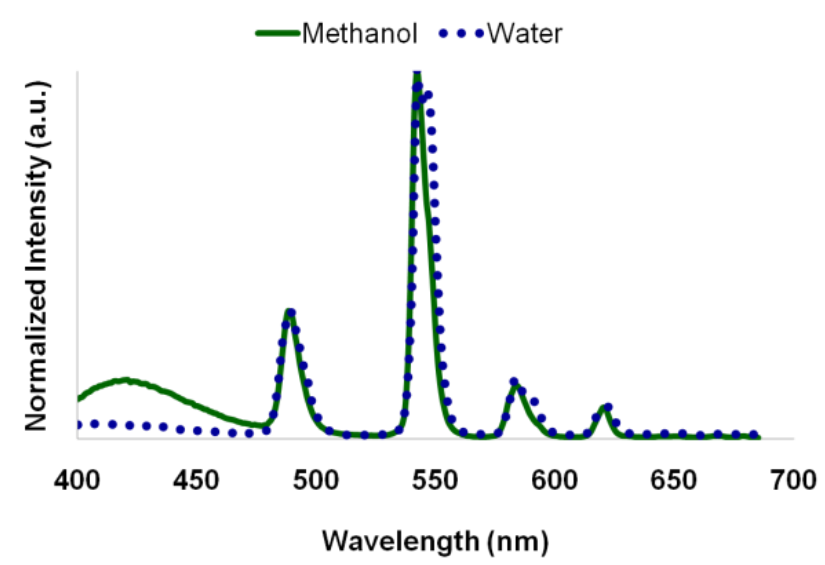

(a)

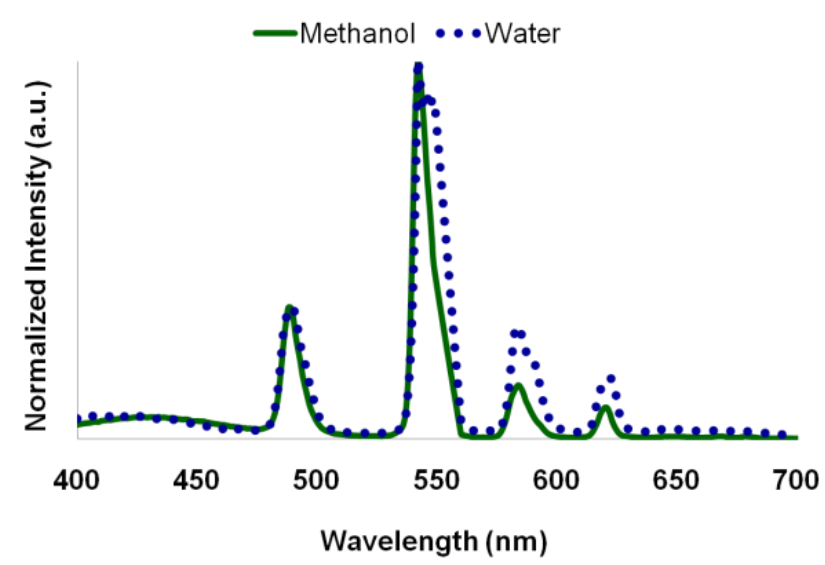

(b)

Organic dyes utilized in photonic devices produce broad emission spectra (FWHM = 50-200 nm) where narrow emission spectra (FWHM $<5 \mathrm{~nm}$ ) are generally preferred [30] for improving color saturation [31] in various light-emission applications such as displays and fashion. The photoluminescence of the polymer nanocomposites synthesized in water and in methanol was measured by normalizing the spectra at $543 \mathrm{~nm}$ and applying Lorentzian fits. The FWHM at the $490 \mathrm{~nm}$ emission peak was computed as it provides insights into the affects of changes in the environment resulting from the ligand and agglomeration [32]. In both cases of direct ion and ligand excitation, the spectral peaks are reasonably narrow ( $\sim 9 \mathrm{~nm}$ for water and $\sim 8 \mathrm{~nm}$ for methanol), though not statistically different given the spectrometer slit-width.

The interaction between temperature and dielectric constant in relationship to the thickness of the electric double layer is key factor in agglomerate size. Water has a relative dielectric constant of $\sim 80$ 
at $25^{\circ} \mathrm{C}$ and $\sim 64$ at $70{ }^{\circ} \mathrm{C}$ and methanol has a dielectric constant of $\sim 33$ at $25{ }^{\circ} \mathrm{C}[33,34]$. The lowering of the dielectric constant of water with the synthesis temperature being elevated to $70{ }^{\circ} \mathrm{C}$ still provides water with a relative dielectric constant that is twice that of methanol at $25{ }^{\circ} \mathrm{C}$. The Debye screening thickness of the nanoparticles synthesized in water should enable the particles to repel one another better than the nanoparticles synthesized in methanol based on the water synthesis maintaining a higher dielectric constant and increased fabrication temperature. Also, the nanoparticles synthesized in methanol should have effectively decreased the electrical double layer between the particles resulting in electrostatic attraction and greater agglomeration. Nanoparticle synthesis performed by Ellerbrock using water as the solvent found that $\mathrm{Tb}^{3+}: \mathrm{LaF}_{3}$ particles without ligand measured $6 \pm 1 \mathrm{~nm}$ in diameter [16]. The average size of the agglomerate diameters as determined by dynamic light scattering for particles synthesized in water and methanol are listed in Table 2.

Table 2. Average agglomerate size of nanoparticles synthesized in water and methanol.

\begin{tabular}{cc}
\hline Synthesis Solvent & Diameter Size by \% Intensity \\
\hline Water & $388 \pm 188 \mathrm{~nm}$ \\
Methanol & $37 \pm 2 \mathrm{~nm}$ \\
\hline
\end{tabular}

Nanoparticle synthesized in methanol produced agglomerates that were, on average, an order of magnitude smaller than the agglomerates produced in water, which is contrary to the above statements and is illustrated in Figure 6. The reduction in size of the agglomerates fabricated in methanol could be attributed to methanol having fewer number of hydrogen bonds per molecule where a stronger ionic interaction between the nanocrystal and the ligand would be induced $[35,36]$.

Figure 6. (a) STEM image of polymer nanocomposite synthesis in water. Inset: agglomerate greater than $200 \mathrm{~nm}$; (b) STEM image of polymer nanocomposite synthesis in methanol.

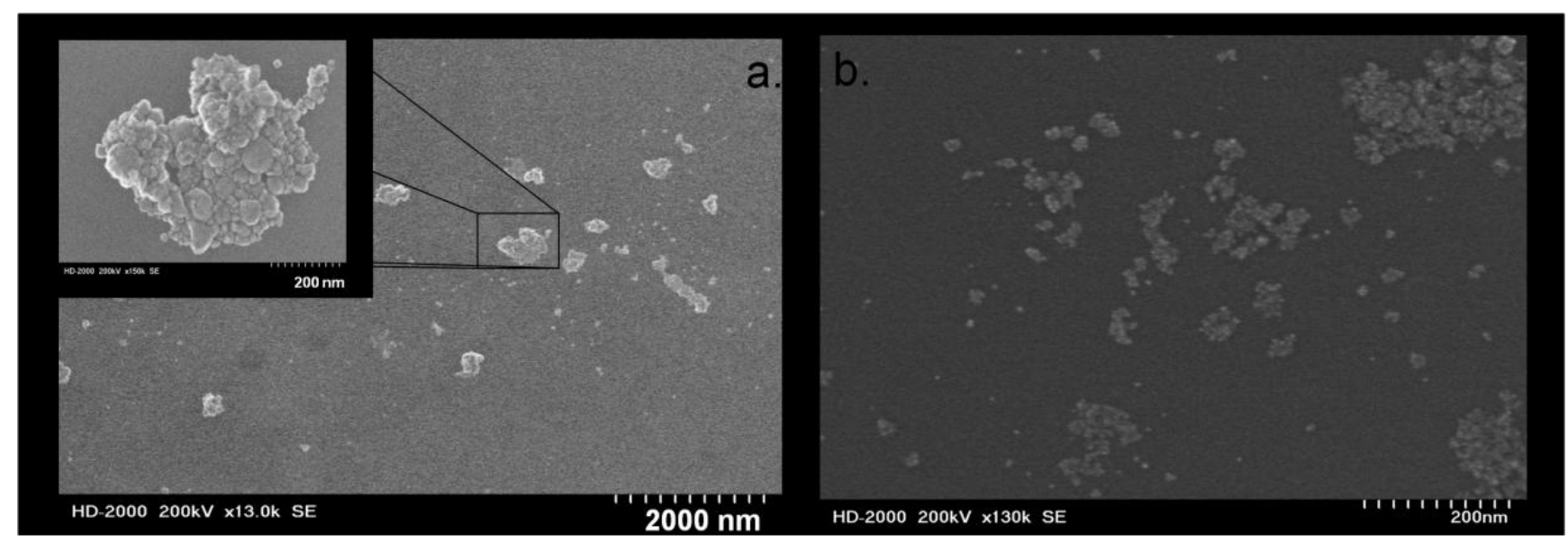

Another factor effecting ionic interaction is $\mathrm{pH}$ as charges on the surface groups can be varied by changing the $\mathrm{pH}$ of the synthesis. Nanoparticles synthesized in water were fabricated at a $\mathrm{pH}$ of 8 where as the $\mathrm{pH}$ of the methanol reaction was not adjusted and was processed at an acidic $\mathrm{pH}$ of 2-3. The methanol synthesis was chosen for further evaluation.

The $\mathrm{pH}$ of the methanol nanoparticle suspension was increased by $1 \mathrm{pH}$ unit starting with a $\mathrm{pH}$ of 3 to a $\mathrm{pH}$ of 8 in Figure 7(a,b), respectively. Figure 7(a) shows the suspension under conventional room 
light and Figure 7(b) displays the luminescence of the nanoparticle suspensions when excited with ultraviolet light at the wavelength of $365 \mathrm{~nm}$. The suspension is clear at the synthesis $\mathrm{pH}$ of 3 . A change in suspension appearance is visually apparent between the synthesis $\mathrm{pH}$ of 3 to 4 and sedimentation occurs where large agglomerates fall out of suspension due to gravity in the suspensions synthesized at $\mathrm{pH} \geq 5$.

Figure 7. (a) Nanoparticle suspensions in methanol synthesized at different $\mathrm{pH}$ under conventional room light; (b) Nanoparticle suspensions in methanol synthesized at different $\mathrm{pH}$ under UV light at wavelength equal to $365 \mathrm{~nm}$.

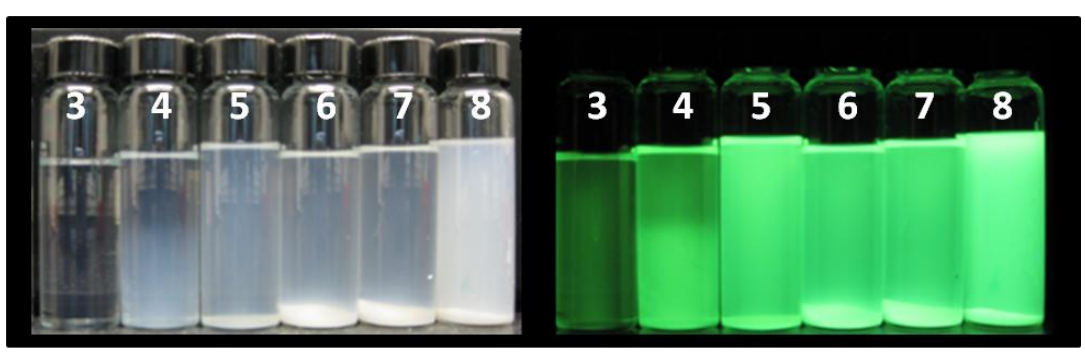

(a)

(b)

Particles displayed positive surface charges in this research as determined by zeta potential measurements, which represents the degree of repulsion between adjacent particles having the same charge [37]. The positive surface charge can be counteracted by the addition of a Lewis base into the suspension which can promote the strengthening of negative charges leading to colloidal instability. Suspensions with zeta potential values between +30 to $-30 \mathrm{mV}$ are considered to be unstable. Zeta potential values below the black dotted line in Figure 8 indicate values below $+30 \mathrm{mV}$; i.e., unstable suspensions. The zeta potential measurements verify the visual analysis of potential agglomerate formation beyond the synthesis $\mathrm{pH}$ of 3 .

Figure 8. Zeta potential of ASA: $\mathrm{Tb}^{3+}: \mathrm{LaF}_{3}$ synthesized in methanol at various $\mathrm{pH}$.

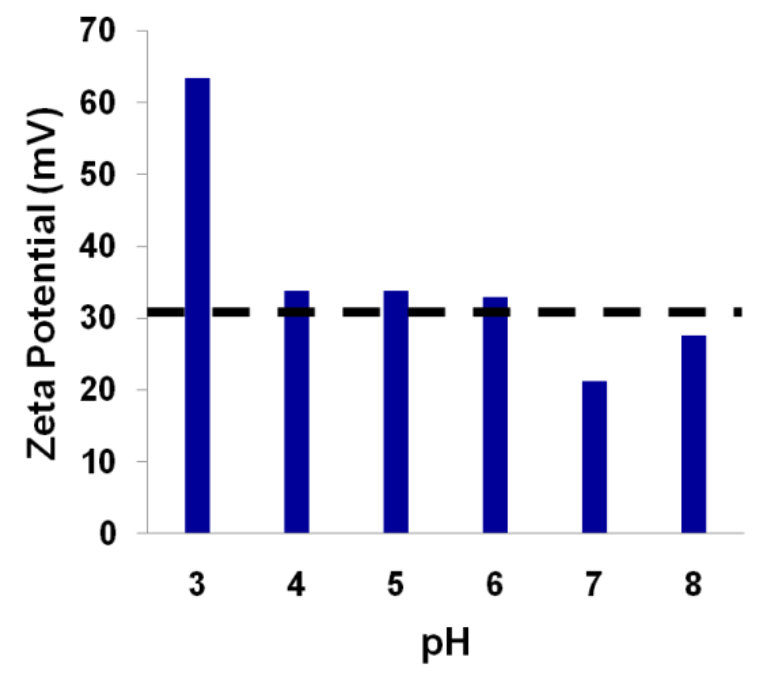

Average agglomerate diameters as determined by dynamic light scattering for the particles synthesized at the various $\mathrm{pH}$ are listed in Table 3. TEM image illustrated in Figure 9 validate the diameter size difference between nanoparticles synthesis at a $\mathrm{pH}$ of 3 as compared to a $\mathrm{pH}$ of 8 . 
Table 3. Average particle size of agglomerates formed in methanol synthesis at various $\mathrm{pH}$.

\begin{tabular}{cc}
\hline $\mathbf{p H}$ & Diameter Size by \% Intensity \\
\hline 3 & $74 \pm 11 \mathrm{~nm}$ \\
4 & $339 \pm 38 \mathrm{~nm}$ \\
5 & $361 \pm 29 \mathrm{~nm}$ \\
6 & $376 \pm 105 \mathrm{~nm}$ \\
7 & $930 \pm 431 \mathrm{~nm}$ \\
8 & $803 \pm 23 \mathrm{~nm}$ \\
\hline
\end{tabular}

Figure 9. (a) TEM image of nanoparticles synthesized in methanol at a $\mathrm{pH}$ of 3; (b) TEM image of nanoparticles synthesized in methanol at a $\mathrm{pH}$ of 8 .

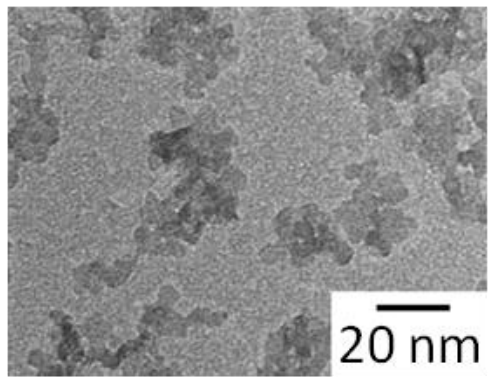

(a)

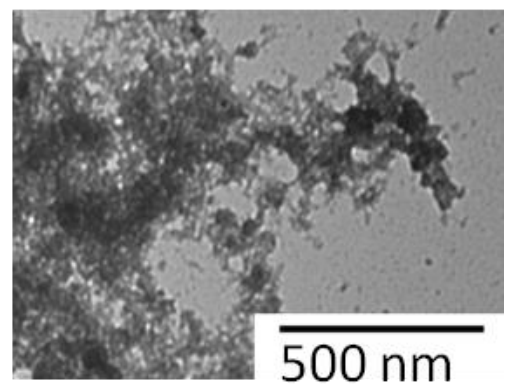

(b)

\section{Experimental Section}

\subsection{Materials}

PMMA $\left(\mathrm{M}_{\mathrm{w}} \approx 130 \mathrm{k}\right.$-Plaskolite West, Inc., Compton, CA, USA) was used as received. Anhydrous tetrahydrofuran, THF (99\%-Acros, Morris Plains, NJ, USA), methanol, MeOH (99.8\%-BDH, West Chester, PA, USA), ultrapure water (18.2 $\mathrm{M} \Omega \mathrm{cm}$, Millipore RiOs and Elix water purification system, Millipore Corporation, Burlington, MA, USA) were used as solvents. Lanthanum (III) nitrate hexahydrate, $\mathrm{La}\left(\mathrm{La}\left(\mathrm{NO}_{3}\right)_{3} \cdot 6 \mathrm{H}_{2} \mathrm{O}, 99.99 \%\right.$ - Sigma-Aldrich, St. Louis, MO, USA) and terbium (III) nitrate hydrate, $\mathrm{Tb}^{3+}\left(\mathrm{Tb}\left(\mathrm{NO}_{3}\right)_{3} \cdot 6 \mathrm{H}_{2} \mathrm{O}, 99.9 \%\right.$-Sigma-Aldrich, St. Louis, MO, USA), acetylsalicylic acid, ASA (MP Biomedicals, LLC, Solon, OH, USA), ammonium fluoride (99.3\%-Fisher Scientific, Fair Lawn, NJ, USA), ammonium hydroxide, $\mathrm{NH}_{4} \mathrm{OH}$ (28-30\% ACS-BDH Aristar-VWR, West Chester, PA, USA), ethanol, EtOH (99.5\%—Acros, Morris Plains, NJ, USA), acetone (99.9\%—BDH, West Chester, PA, USA) also were used as received.

\subsection{Nanoparticle Synthesis}

$\mathrm{Tb}^{3+}: \mathrm{LaF}_{3}$ nanocrystals were prepared in aqueous solutions with water as the solvent for the water process and methanol as the solvent for the methanol process. The water and methanol synthesis processes are depicted in Figure 10(a,b) respectively. The rare-earth solution of $\mathrm{La}\left(\mathrm{NO}_{3}\right)_{3} \cdot 6 \mathrm{H}_{2} \mathrm{O}$ $(9 \mathrm{mmol})$ and $\mathrm{Tb}\left(\mathrm{NO}_{3}\right)_{3} \cdot 6 \mathrm{H}_{2} \mathrm{O}(2 \mathrm{mmol})$ in $16 \mathrm{~mL}$ of solvent, water and methanol respectively, was prepared at room temperature. $\mathrm{NH}_{4} \mathrm{OH}$ was added to the rare-earth solution in water to adjust the solution to $\mathrm{pH}$. The $\mathrm{pH}$ of the methanol solution was not adjusted. 
Figure 10. (a) Flowchart nanoparticle synthesis in water; (b) Flowchart nanoparticle synthesis in methanol.

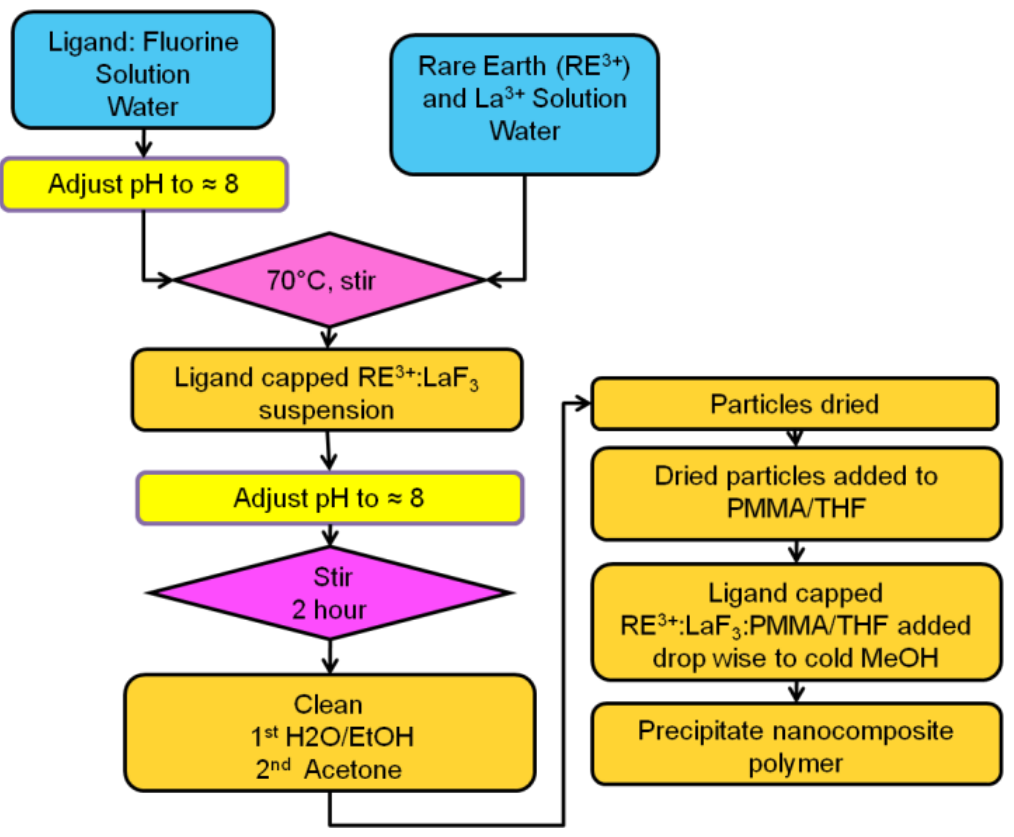

(a)

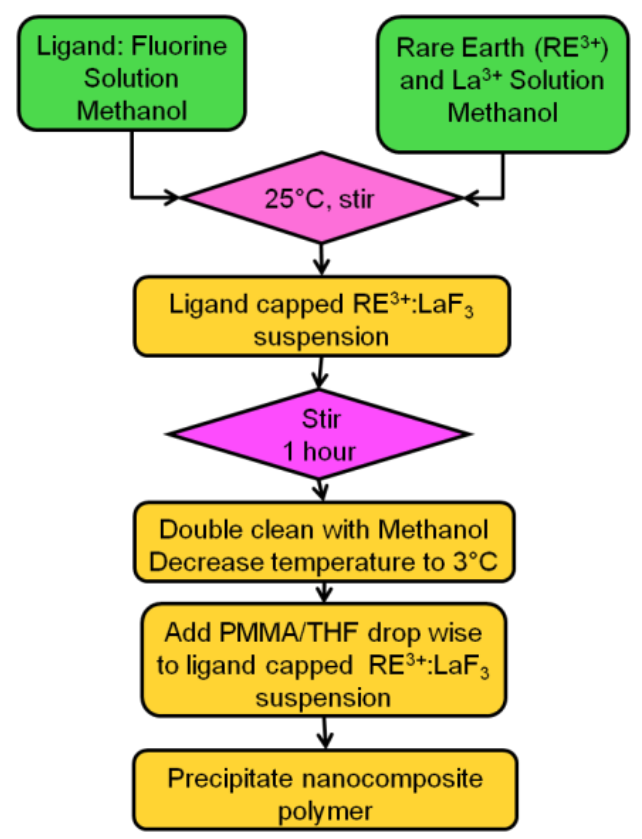

(b)

Water Synthesis: The rare-earth solution in water was added drop-wise into a stirred solution of $\mathrm{NH}_{4} \mathrm{~F}$ $(11 \mathrm{mmol})$ and ASA $(20 \mathrm{mmol})$ in water at $70{ }^{\circ} \mathrm{C}$. The volume ratio of water/ASA was 40:1. $\mathrm{NH}_{4} \mathrm{OH}$ was added to the suspension to adjust the solution to $\mathrm{pH}$ 8. The suspension was stirred for $2 \mathrm{~h}$ at $70{ }^{\circ} \mathrm{C}$. A centrifuge was used to separate the precipitate at a setting of 3,000 rpm for $10 \mathrm{~min}$. The nanoparticles produced from the water suspension were washed with $50 \mathrm{vol} \%$ of EtOH in water followed by an acetone wash. Synthesized particles were dried over night in a vacuum oven and then added to 5 wt $\%$ of PMMA in THF to form the polymer nanoparticle suspension, ASA: $\mathrm{Tb}^{3+}: \mathrm{LaF}_{3}: \mathrm{PMMA}_{\mathrm{THF}}$. $\mathrm{ASA}: \mathrm{Tb}^{3+}: \mathrm{LaF}_{3}: \mathrm{PMMA} / \mathrm{THF}$ was added drop wise to excess (v/v of suspension to $\mathrm{MeOH} ; 1: 400$ ) stirred $\mathrm{MeOH}$ at $\sim{ }^{\circ} \mathrm{C}$. The product was precipitate nanocomposite polymer powder.

Methanol Synthesis: The rare-earth solution in methanol was added drop-wise into a stirred solution of $\mathrm{NH}_{4} \mathrm{~F}(12 \mathrm{mmol})$ and $\mathrm{ASA}(23 \mathrm{mmol})$ in methanol at room temperature, $\sim 25{ }^{\circ} \mathrm{C}$. The volume ratio of methanol/ASA was 40:1. The $\mathrm{pH}$ was not adjusted. The suspension was stirred for $1 \mathrm{~h}$. A centrifuge was used to separate the precipitate at a setting of 3,000 rpm for $10 \mathrm{~min}$. Methanol synthesized particles were washed by a two-cycle neat methanol wash. Neat $\mathrm{MeOH}$ was added to the suspension and the temperature of the stirred suspension was decreased to approximately $3{ }^{\circ} \mathrm{C}$. After 30 min, a solution of $5 \mathrm{wt} \%$ of PMMA in THF was added drop wise to the ASA: $\mathrm{Tb}^{3+}: \mathrm{LaF}_{3}: \mathrm{MeOH}$ suspension at a v/v of 1:400. The product was precipitate nanocomposite polymer powder.

The precipitate nanocomposite polymer powder was vacuum filtered, washed in $\mathrm{MeOH}$ and then dried under vacuum for over $12 \mathrm{~h}$. 


\subsection{Characterization}

The composition of the nanoparticles were characterized utilizing a scanning transmission electron microscope (STEM, Hitachi HD2000, Pleasanton, CA, USA) equipped with an Oxford INCA Energy 200 Energy Dispersive Spectrometer (EDX).

Optical absorption was performed using a Perkin Elmer Lambda 900 UV-Vis-NIR Spectrometer (Waltham, MA) with UV Winlab Version 3.00 .03 software. A $1 \mathrm{~nm}$ slit size in the UV and visible range of 260-380 $\mathrm{nm}$ was used to perform the scans. Samples were prepared by dispersing the ligand into water and methanol where all measurements were done at room temperature.

A Jobin-Yvon Fluorolog Tau 3 Fluorometer (Edison, NJ, USA) with $4 \mathrm{~nm}$ emission bandpass was used for emission spectra measurements and $1 \mathrm{~nm}$ slit width was used for excitation spectra measurements. $1 \mathrm{~nm}$ intervals with $50 \mathrm{~ms}$ integration time was used to collect the data. Measurements were performed at room temperature. Lorentzian curve fits correcting for a constant background in Igor Pro 6.1 (Wavemetrics, Portland, OR, USA) were superimposed on excitation spectra for full-width at half maximum (FWHM) measurements.

Zetasizer Nano Series Nano ZS-Dynamic Light Scattering (DLS) module from Malvern (Worcestershire, UK) was utilized to measure the size and size distribution of particles at room temperature. Nanoparticles fabricated in water were ball milled using Teflon ${ }^{\circledR}$ spheres into a fine powder before resuspension in neat water. The liquid suspension of nanoparticles in methanol was also measured. The average of three samples was used to determine the size distributions.

Electron micrographs were obtained using a STEM and a transmission electron microscope (TEM, Hitachi H7600T, Pleasanton, CA, USA). Samples were prepared by drop casting nanoparticle suspensions followed by subsequent solvent evaporation onto a 200 mesh carbon coated copper TEM grids.

A Brookhaven (Holtsville, NY, USA) Zeta Plus-Zeta Potential Analyzer was used to determine surface charge of nanoparticles. The stock solution was prepared by dissolving $0.037 \mathrm{~g}$ of potassium chloride $(\mathrm{KCl})$ into $500 \mathrm{~mL}$ of ultrapure water to create a concentration $1 \mathrm{mM}$. $200 \mathrm{~mL}$ of the stock solution was used and stirred during evaluation. The starting $\mathrm{pH}$ of stock solution was adjusted with hydrochloric acid in water $(4.0 \mathrm{M})$ to reach a $\mathrm{pH}$ of 3 . The sample of $24 \mathrm{~mL}$ of nanoparticles synthesized and suspended in methanol at a $\mathrm{pH}$ of 3 was added to the stock solution. A solution of sodium hydroxide at $0.1 \mathrm{M}$ concentration was used to adjust the stock solution $\mathrm{pH}$.

\section{Conclusions}

Factors that affect the particles ability to agglomerate based on the electrical double layer equation were investigated. In order to verify the inclusion of the optically active nanocrystals into transparent PMMA the molar ratio of $\mathrm{La}^{3+}$ to $\mathrm{Tb}^{3+}$ was confirmed by EDX analysis to be on average 5:1 and 2:1 for nanoparticles synthesized with acetylsalicylic acid in water and methanol, respectively.

Energy transfer from the ligand to the RE ion was concluded by measuring the maximum absorption of the ligand using UV-Vis absorption spectroscopy. ASA in water and in methanol produced a maximum absorption peak at $276 \mathrm{~nm}$. Characteristic peaks of $\mathrm{Tb}^{3+}$ existed in the emission spectra of nanoparticles synthesized in water and methanol upon direct ion and ligand excitation. 
Nanoparticle synthesized in methanol produced agglomerate diameters about twelve times smaller than the agglomerates diameters produced in water. The methanol synthesis was chosen for further evaluation of the effect of $\mathrm{pH}$ on the agglomeration.

Nanoparticles synthesized in methanol have a positive surface charge and at a $\mathrm{pH}$ of 3 the suspension is transparent. A visual change in the suspension becomes apparent at a pH of 4 . Sedimentation begins and continues at the $\mathrm{pH}$ range of 5 to 8 . DLS analysis confirmed the agglomerate size of the nanoparticles synthesized at a $\mathrm{pH}$ of 3 to be on average $74 \mathrm{~nm}$. Nanoparticles synthesized at a pH of 4 or higher produced agglomerates greater than the direct ion and ligand excitation wavelengths.

\section{Acknowledgments}

We acknowledge the support of the National Textile Center through grant award M10-CLO5. Fellowship funding at the time of this work was provided by the South East Alliance for Graduate Education and the Professoriate (SEAGEP) on the National Science Foundation award HRD-0450279, Center of Optical Materials Science and Engineering Technologies (COMSET) and School of Materials Science and Engineering at Clemson University. A note of gratitude is given to Gregory Von White II, Julien Boyon, Cody Reynolds and Textile 421 class for their help with this project.

\section{References}

1. Klink, S.I.; Hebbink, G.A.; Grave, L.; Peters, G.A.; van Veggel, F.C.J.M.; Reinhoudt, D.N.; Hofstraat, J.W. Near-infrared and visible luminescence from terphenyl-based lanthanide(III) complexes bearing amido and sulfonamido pendant arms. Eur. J. Org. Chem. 2000, 2000, 1923-1931.

2. Ziemann, O.; Poisel, H.; Randel, S.; Lee, J. Polymer Optical Fibers for Short, Shorter and Shortest Data Links. In Proceedings of OFC/NFOEC 2008, 2008 Conference on Optical Fiber Communication/National Fiber Optic Engineers Conference, San Diego, CA, USA, 24-28 February 2008.

3. Jiu, H.; Zhang, L.; Liu, G.; Fan, T. Fluorescence enhancement of samarium complex co-doped with terbium complex in a poly(methyl methacrylate) matrix. J. Lumin. 2009, 129, 317-319.

4. Zubia, J.; Arrue, J. Plastic optical fibers: An introduction to their technological processes and applications. Opt. Fiber Technol. 2001, 7, 101-140.

5. Sharma, P.K.; Nass, R.; Schmidt, H. Effect of solvent, host precursor, dopant concentration and crystallite size on the fluorescence properties of Eu(III) doped yttria. Opt. Mater. 1998, 10, 161-169.

6. Bekiari, V.; Lianos, P. Multicolor emission from terpyridine-lanthanide ion complexes encapsulated in nanocomposite silica/poly(ethylene glycol) sol-gel matrices. J. Lumin. 2003, 101, 135-140.

7. Brunet, E.; Juanes, O.; Rodriguez-Ubis, J. Supramolecularly organized lanthanide complexes for efficient metal excitation and luminescence as sensors in organic and biological applications. Curr. Chem. Biol. 2007, 1, 11-39.

8. Kuriki, K.; Koike, Y.; Okamoto, Y. Plastic optical fiber lasers and amplifiers containing lanthanide complexes. Chem. Rev. 2002, 102, 2347-2356. 
9. Maji, S.; Viswanathan, K.S. Ligand-sensitized fluorescence of $\mathrm{Eu}^{3+}$ using naphthalene carboxylic acids as ligands. J. Lumin. 2008, 128, 1255-1261.

10. Li, W.; Zheng, Y.; Sun, X.; Shi, X.; Chai, W.; Ren, T. Synthesis and fluorescence properties of lanthanide (III) perchlorate complexes with naphthyl-naphthalinesulphonylpropyl sulfoxide. J. Fluoresc. 2010, 20, 235-241.

11. Sun, X.; Li, W.; Chai, W.; Ren, T.; Shi, X. The studies of enhanced fluorescence in the two novel ternary rare-earth complex systems. J. Fluoresc. 2010, 20, 453-461.

12. Velsko, S.P.; Eimerl, D. Second-harmonic generation in sodium lanthanum fluoride. J. Appl. Phys. 1987, 62, 2461-2465.

13. Kumar, G.A.; Chen, C.W.; Ballato, J.; Riman, R.E. Optical characterization of infrared emitting rare-earth-doped fluoride nanocrystals and their transparent nanocomposites. Chem. Mater. 2007, 19, 1523-1528.

14. Chen, D.; Yu, Y.; Huang, P.; Wang, Y. Nanocrystallization of lanthanide trifluoride in an aluminosilicate glass matrix: Dimorphism and rare earth partition. CrystEngComm 2009, 11, 1686-1690.

15. Luo, Y.; Yu, X.; Su, W.; Zhang, Z.; Wu, W.; Yan, Q.; Zhang, Q. Energy transfer in a ternary system composed of $\mathrm{Tb}(\mathrm{DBM})_{3} \mathrm{Phen}, \mathrm{Eu}(\mathrm{DBM})_{3} \mathrm{Phen}$, and poly(N-vinylcarbazole). J. Mater. Res. 2009, 24, 3023-3031.

16. Ellerbrook, B.M. Fabrication of Fluorescent Nanoparticle-Polymer Composites for Photoactive-Based Materials. M.Sc. Thesis, Clemson University, Clemson, SC, USA, 2009.

17. Jacobsohn, L.G.; Sprinkle, K.B.; Kucera, C.J.; James, T.L.; Roberts, S.A.; Qian, H.; Yukihara, E.G.; DeVol, T.A.; Ballato, J. Synthesis, luminescence and scintillation of rare earth doped lanthanum fluoride nanoparticles. Opt. Mater. 2010, 33, 136-140.

18. Helmholtz, H. Ueber einige gesetze der verteilung elektrischer ströme in körperlichen leitern mit anwendung auf die thierisch-elektrischen versuche. Ann. Phys. Chem. 1853, 165, 211-233.

19. Gouy, G. About the electric charge on the surface of an electrolyte. J. Phys.Theor. Appl. 1910, 9 , 457-468.

20. Gouy, G. About the electric charge on the surface of an electrolyte. Compt. Rend. 1909, 149, 654-657.

21. Chapman, D.L. A contribution to the theory of electrocapillarity. Philos. Mag. 1913, 25, 475-481.

22. Stern, O. Zur theorie der elektrischen Doppelschicht. Zeitschrift fur Electrochemie 1924, 30, 508-516.

23. Ravina, L. Everything You Want to Know about Coagulation \& Flocculation [Guide]; Zeta-Meter, Inc.: Staunton, VA, USA, 1993; Volume 4, pp. 1-41.

24. Hiram, Y.; Nir, A. A simulation of surface tension driven coalescence. J. Colloid Interface Sci. 1983, 95, 462-470.

25. Hu, H.; Chen, Z.; Cao, T.; Zhang, Q.; Yu, M.; Li, F.; Yi, T.; Huang, C. Hydrothermal synthesis of hexagonal lanthanide-doped $\mathrm{LaF}_{3}$ nanoplates with bright upconversion luminescence. Nanotechnology 2008, 19, 375702.

26. Kokuoz, B.; Kucera, C.; DiMaio, J.R.; Ballato, J. Organic-inorganic hybrid nanoparticles with enhanced rare-earth emissions. Opt. Mater. 2009, 31, 1327-1330. 
27. Gutzov, S.; Bredol, M. Preparation and luminescence of terbium and cerium-doped silica xerogels. J. Mater. Sci. 2006, 41, 1835-1837.

28. Pinna, N. The "benzyl alcohol route": An elegant approach towards organic-inorganic hybrid nanomaterials. J. Mater. Chem. 2007, 17, 2769-2774.

29. Sun, Y.; Qi, L.; Lee, M.; Lee, B.I.; Samuels, W.D.; Exarhos, G.J. Photoluminescent properties of $\mathrm{Y}_{2} \mathrm{O}_{3}: \mathrm{Eu}^{3+}$ phosphors prepared via urea precipitation in non-aqueous solution. J. Lumin. 2004, 109, 85-91.

30. Diaz-Garcia, M.A.; De Avila, S.F.; Kuzyk, M.G. Energy transfer from organics to rare-earth complexes. Appl. Phys. Lett. 2002, 81, 3924-3926.

31. Fairchild, M.D. Color Appearance Models: CIECAM02 and Beyond. In Proceedings of IS\&T/SID 12th Color Imaging Conference, Scottsdale, AZ, USA, November 2004; pp. 1-68.

32. Selvin, P.R.; Hearst, J.E. Luminescence energy transfer using a terbium chelate: Improvements on fluorescence energy transfer. Proc. Natl. Acad. Sci. USA 1994, 91, 10024-10028.

33. Newton, B. $\mathrm{O}=\mathrm{CHem}$ : Solvents. Available online: http://www.usm.maine.edu/ newton/ Chy251_253/Lectures/Solvents/Solvents.html (accessed on 16 July 2010).

34. Lorenz, K.J.; Kulp, K. Book review: Handbook of cereal science and technology. Food Bioprod Process.: Trans. Inst. Chem. Eng. Part C 1996, 74, 244.

35. Yun, S.S.; Choi, K.Y.; Park, K.K.; Suh, I.S. Luminescence study on some terbium (III) complexes in water and water-ethanol mixtures. Bull. Korean Chem. Soc. 1985, 6, 33-36.

36. Gulmen, T.S.; Sibert, E.L., III. Vibrational energy relaxation of the $\mathrm{OH}$ stretch in liquid methanol. J. Phys. Chem. A 2004, 108, 2389-2401.

37. Raj, S.P.; Angela, L.S. Stability of marketed albendazole suspensions and correlation between zeta potential and sedimentation. Int. Res. J. Pharm. 2011, 3, 154-156.

(C) 2011 by the authors; licensee MDPI, Basel, Switzerland. This article is an open access article distributed under the terms and conditions of the Creative Commons Attribution license (http://creativecommons.org/licenses/by/3.0/). 